Atehortúa, T.; Mejía, L. (2018). Tipos de decisiones con base en las herramientas de contabilidad de gestión en las empresas de confección. Contaduría Universidad de Antioquia, 72, 107-129

Doi: https://doi.org/10.17533/udea.rc.n72a06

\title{
Tipos de decisiones con base en las herramientas de contabilidad de gestión en las empresas de confección
}

\author{
Tania Atehortúa Castrillón \\ tania.atehortua@udea.edu.co \\ Universidad de Antioquia \\ Laura Cristina Mejía Valencia \\ laura.mejiav@udea.edu.co \\ Universidad de Antioquia
}


Tipos de decisiones con base en las herramientas de contabilidad de gestión en las empresas de confección

Resumen: La Contabilidad de gestión está cada vez más posicionada dentro de las empresas para que éstas logren ventajas competitivas que les permitan desarrollo y crecimiento tanto a nivel nacional, como internacional. El trabajo de investigación busca determinar el tipo de decisiones que se toman con base a las distintas herramientas de contabilidad de gestión en las empresas de confección del municipio de La Ceja, Antioquia (Colombia), es decir, herramientas para mejorar la gestión y la planeación estratégica, evaluando beneficios como la eficiencia, la productividad, la efectividad y la satisfacción del cliente que ayuden a mejorar internamente la cadena de valor y que además aporte al desarrollo continuo de la organización.

Palabras clave: Contabilidad de gestión, herramientas de contabilidad de gestión, toma de decisiones, empresas de confección, tipos de decisiones.

Types of decisions based on management accounting tools in apparel companies

Abstract: Management accounting is increasingly more positioned in companies, allowing them to obtain competitive advantages to develop and grow at national and international level. This research work seeks to determine the type of decisions made based on the different management accounting tools in apparel companies of the municipality of La Ceja, Antioquia (Colombia). Those are tools to improve strategic management and planning, evaluating benefits such as efficiency, productivity, effectiveness and customer satisfaction, which help improve the value chain internally and also contribute to the continuous development of the organization.

Keywords: Management accounting, management accounting tools, decision making, apparel companies, types of decisions.

Types de décisions fondées sur les outils de comptabilité de gestion dans les entreprises de confection de vetêments

Résumé: La comptabilité de gestion est de plus en plus positionnée à l'intérieur des entreprises afin d'obtenir des avantages concurrentielles permettant le devéloppement et la croissance nationale et internationale. Ce travail de recherche vise à déterminer le type de décisions prises selon les differents outils de comptabilité de gestion dans les entreprises de confection de vetêments à La Ceja, ville d'Antioquia, en Colombie. Des outils d'amélioration de la gestion et de la planification stratégique, en tenant compte des avantages telles que l'efficacité, la productivité, l'effectivité et la satisfaction du client. Ces outils doivent agir aussi à l'intérieur de la chaîne de valeur ainsi que sur le devéloppement permanente de l'organisation.

Mots-clés: Comptabilité de gestion, outils de comptabilité de gestion, prise de décisions, entreprise de confection de vetêments, types de décisions.

Tipos de decisões baseadas nas ferramentas de contabilidade de gestão nas empresas de confecção

Resumo: A contabilidade de gestão está cada vez mais posicionada no interior das empresas para que estas obtenham vantagens competitivas que lhes permita o desenvolvimento e o crescimento tanto nacional quanto internacionalmente. O trabalho de pesquisa pretende determinar o tipo de decisões que se tomam com base nas diferentes ferramentas de contabilidade de gestão nas empresas de confecção do município "La Ceja”, Antioquia (Colômbia), ou seja, ferramentas para melhorar a gestão e o planejamento estratégico, avaliando beneficios como a eficiência, a produtividade, a efetividade e a satisfação do cliente que ajudem a melhorar internamente a cadeia de valor e que também aporte ao desenvolvimento contínuo da organização.

Palavras chave: Contabilidade de gestão, ferramentas de contabilidade de gestão, toma de decisões, empresas de confecção, tipos de decisões. 
Cont. udea (enero-junio), pp. 107-129. (C) Universidad de Antioquia-2018.

\title{
Tipos de decisiones con base en las herramientas de contabilidad de gestión en las empresas de confección
}

\author{
Tania Atehortúa Castrillón y Laura Cristina Mejía Valencia
}

Doi: https://doi.org/10.17533/udea.rc.n72a06

Primera versión recibida en mayo de 2018- Versión final aceptada en junio de 2018

\section{Introducción}

$\mathrm{Cl}^{\mathrm{l}}$ ambiente empresarial actual, crea la necesidad a las empresas de tener un Esistema de gestión completo que les permita tener una mayor eficiencia para que las mismas se adapten al mundo cambiante en el que se encuentran inmersas aumentando sus posibilidades de crecimiento en el mercado, pues se concibe hoy la organización como un sistema que relaciona varios subsistemas que interactúan entre sí.

En coherencia con lo que exponen Buelvas y Mejía (2015) "el mundo tiene una dinámica evolutiva muy fuerte" (p. 92); existen factores internos como externos que afectan el flujo normal de las organizaciones, por ello, las empresas deben tomar conciencia sobre la necesidad de evolucionar y tomar decisiones que conlleven a esto. Por ello, es necesario buscar prácticas contables que complementen los sistemas tradicionales, para que promuevan el mejoramiento continuo en el desarrollo de la actividad empresarial, consecuente con el éxito en la toma de decisiones ante la incertidumbre del mercado, para lo cual deben tener información completa y confiable, logrando parte de esto con la utilización de herramientas contables y gerenciales que apoyen dicha toma de decisiones en las organizaciones.

El objetivo del trabajo de investigación, es determinar el tipo de decisiones basadas en las herramientas de contabilidad de gestión que toman las empresas del sector de la confección del municipio de La Ceja.

El artículo se compone de un acápite teórico que permite al lector relacionarse con el concepto de contabilidad de gestión y su importancia en las empresas de confección, el aporte que le pueden brindar las herramientas 
Atehortúa, T.; Mejía, L. Tipos de decisiones con base en las herramientas de contabilidad...

gerenciales, entre otros; además se presentan los resultados a través del análisis de la información obtenida a partir del instrumento utilizado, donde se puede evidenciar las herramientas de la contabilidad de gestión y su uso en la toma de decisiones.

La investigación es de tipo cualitativo debido a que el trabajo busca conocer un fenómeno a profundidad a través de un proceso inductivo, es decir, se exploró la situación de las empresas de confección del municipio de La Ceja para analizar el tipo de decisiones que toman éstas organizaciones con base a la utilización de herramientas de la contabilidad de gestión.

\section{Análisis del contexto}

\section{II.1 El sector de la confección}

La actividad económica colombiana está dividida en sectores económicos de acuerdo con los procesos productivos que se desarrollan en ellos; según la economía clásica, en Colombia se encuentran el sector primario o sector agropecuario, sector secundario o sector industrial y el sector terciario o sector de servicios(Red Cultural del Banco de la República en Colombia, 2000).

Las empresas dedicadas a la confección se encuentran en el sector industrial, ya que se dedican a la transformación de materia prima para la consecución de un producto final; este sector es uno de los que mayor participación tiene en el mercado internacional y a su vez ha aportado en gran medida al crecimiento y desarrollo del País. La clasificación de las empresas de confección está enfocada principalmente en la Ley 905 del 02 de agosto de 2004, según el Artículo $2^{\circ}$ de dicha norma se pueden clasificar las empresas según los siguientes rangos:

Tabla 1. Clasificación de las empresas

\begin{tabular}{lll}
\hline \multicolumn{1}{c}{ Clasificación } & \multicolumn{1}{c}{ Planta de personal } & \multicolumn{1}{c}{ Activos totales } \\
\hline Microempresa & No superior a 10 empleados & Menor a 500 SMMLV \\
Pequeña empresa & Entre 11 y 50 empleados & Entre 501 y 5.000 SMMLV \\
Mediana empresa & Entre 51 y 200 empleados & Entre 5.001 y 30.000 SMMLV \\
\hline
\end{tabular}

Fuente: elaboración propia basada en Ley 905 del 2004.

En el municipio de La Ceja se encuentran diversos tipos de empresas dedicadas al sector de la confección, éstas son: las empresas que se dedican únicamente al ensamble de prendas, es decir, que trabajan para terceros, comunmente conocidas como maquilas; las empresas que se dedican sólo a la comercialización y las empresas que realizan ambas funciones, es decir, se dedican a la producción y a la comercialización de las prendas.

El municipio de La Ceja cuenta con 32 Micro, Pequeñas y Medianas empresas (MIPYMES), las cuales hacen parte del motor de desarrollo de la región y el sustento de la población. Éstas MIPYMES son consideradas como una gran 
fortaleza para el desarrollo económico de la región, por lo que es necesario que estén bien organizadas y dirigidas, así como requieren de un equipo de trabajo capacitado tanto a nivel productivo, financiero y administrativo, para que en la toma de decisiones se tengan criterios claros y estén bien documentados e informados sobre el estado actual de la empresa en todas sus áreas.

Teniendo en cuenta que el municipio de La Ceja es de gran importancia para la región del Oriente Antioqueño, pues muchas familias tienen un desarrollo sostenible a través de éste, se considera necesario organizar todos los procesos y actividades, para que la economía de la región tenga crecimiento y las empresas permanezcan a lo largo del tiempo.

El grado de informalidad en las empresas de confección del municipio de La Ceja es muy alto, y las pocas empresas que están legalmente constituidas no cuentan con la información necesaria para administrar sus recursos, por tal motivo el contador en varias de éstas organizaciones es el único que tiene el conocimiento profesional y debe establecer algunas herramientas gerenciales que le permitan apoyar el direccionamiento de la empresa en busca de la rentabilidad y el aprovechamiento de los recursos.

En coherencia, es importante fortalecerlo mediante indicadores de gestión para que logre altos niveles de competitividad y mejor desempeño en el mercado, en concordancia García y Pérez (2015), plantean que las MYPIMES no cuentan con el conocimiento necesario para entender la importancia de la contabilidad en función a su utilidad además señalan que la contabilidad tiene un rol muy importante para el funcionamiento de la empresa, puesto que le permite planear y proponer objetivos, que le ayuden a elevar los niveles de competitividadcon ayuda de la Responsabilidad Social Empresarial que consideran además fundamental para el desarrollo de la empresa; es entonces la gestión contable la que infiere directamente en la toma de decisiones organizacionales.

\section{II.2 La contabilidad de gestión y su importancia en las organizaciones}

La contabilidad de gestión es una rama de la contabilidad que permite la planeación estratégica en las organizaciones y por ende el buen desempeño de la dirección para la consecución de los objetivos de la organización, en coherencia, Fernández (1994) plantea como definición que:

La Contabilidad de gestión en el contexto de la excelencia empresarial y dentro del área de control total de la calidad, permitirá el conocimiento de variables financieras y no financieras, cuantitativas y cualitativas, fundamentales para conseguir el éxito de la gestión. (Fernández, 1994, p. 869)

Reafirmando esta definición López explica que: 
Atehortúa, T.; Mejía, L. Tipos de decisiones con base en las herramientas de contabilidad...

Debe señalarse que en la actualidad los factores críticos de éxito, no sólo están relacionados con la minimización de los costos, sino también con la satisfacción del cliente, la calidad, la flexibilidad, el mercado, etc., lo cual hace necesario tomar en consideración no sólo factores financieros, sino también indicadores cualitativos, tanto internos (satisfacción al cliente, calidad, plazos de entrega, productividad) como externos (competencia, innovaciones tecnológicas, cambios en la legislación). (López, 2000, p. 47)

Las características que describen estos autores de la contabilidad de Gestión, muestran que es un sistema integral que contrarresta la inflexibilidad de la Contabilidad Financiera, el sistema contable está compuesto por la contabilidad financiera, la contabilidad de costos y la contabilidad gerencial y así mismo explica que entre la contabilidad financiera y la contabilidad gerencial existe una conexión llamada contabilidad de costos, es decir, los conceptos y técnicas de ésta se utilizan tanto en la contabilidad financiera como en la contabilidad gerencial. (Cuevas, 2001)

La importancia de la contabillidad de gestión es reconocida por los contadores públicos y algunos gerentes que con ayuda de las herramientas gerenciales, embarcan a las organizaciones en busca de la rentabilidad, así como lo menciona Quesada (2007) "nuevas técnicas y herramientas logran sustituir las ya tradicionales, en aras de establecer el máximo de eficiencia en la gestión empresarial y para ello se introducen nuevos procesos, encaminados a fortalecer internamente a la organización con respecto al entorno" (p.7)y a las exigencias del mercado.

Es importante resaltar que la contabilidad de gestión se ha venido apoyando de la contabilidad de costos, ya que esta última revela y reporta información financiera y monetaria y a su vez como lo dice Osorio, Ripoll, Álvarez y Atehortúa (2016) "puede ofrecer los diferentes sistemas de costeo que permiten tener información parcial de base para diferentes cuestiones organizacionales como precios, rentabilidad, productividad, racionalización” (p. 132). La información de la contabilidad de costos por si sola no es un medio para realizar una adecuada gestión estratégica, las organizaciones deben tener un respaldo de la información cuantitativa que les brinda la contabilidad de costos, lo cual es afirmado por Osorio, Ripoll, Álvarez y Atehortúa al establecer que es necesario implementar

Sistemas de Gestión de Costos en las empresas, de modo que toda aquella información reportada por los costos tenga un respaldo no solamente cuantitativo, sino también cualitativo, lo que permite así que la administración tenga criterios más sólidos para tomar decisiones. (Osorio, Ripoll, Álvarez y Atehortúa, 2016, p. 132)

Las transformaciones que vienen presentando las empresas, inducen a que la contabilidad también se desarrolle, las empresas se encuentran inmersas en una complejidad organizacional que ha hecho que la contabilidad de costos 
evolucione, dando paso a la contabilidad de gestión entre la que se encuentra la contabilidad de costos actualmente y como su evolución más alta se tiene la contabilidad de dirección estratégica, como un nuevo paradigma contable que busca la generación de información para así lograr una adaptación más rápida y eficaz de las empresas en el nuevo entorno organizacional (Chacon, 2007).

En las empresas, el despliegue interno de información genera cambios con la misma velocidad que el mercado. Cuando la información no es lo suficientemente precisa para la operación que se necesita realizar, se incrementan los costos de producción, se realizan operaciones innecesarias, se genera pérdida de tiempo, de productividad, de rentabilidad y competitividad, lo que se traduce en ineficiencia; por estos motivos las empresas pueden correr el riesgo de salir del mercado.

Todos estos problemas se pueden evitar o mitigar con la utilización de herramientas de contabilidad de gestión que se acomoden específicamente a lo que necesita la empresa, pues esta rama de la contabilidad apoyada en otras tales como: los costos, la reingeniería de procesos, las finanzas y el control pueden contribuir para que las empresas de confección del municipio de La Ceja aumenten su rentabilidad vía productividad, eficiencia y calidad en todos sus procesos y actividades tanto a nivel administrativo como productivo.

Entre los objetivos básicos que persigue la contabilidad de gestión se encuentran, analizar los costos, suministrar información necesaria para las operaciones de planificación, evaluación y control, participando en la toma de decisiones estratégicas, tácticas y operativas y contribuir de esta forma a coordinar la organización.

Tal como afirma Di Ranni (2016):

El sistema contable de gestión hace posible el suministro de información que asegura el monitoreo de todas las funciones de la organización. La Contabilidad de gestión, proveerá la información que hará posible la mejora de la eficiencia y consecuentemente la mejora del resultado económico del ejercicio. (p. 223)

Además, este autor continúa aclarando que con la evolución de la contabilidad de gestión se podrá monitorear cada una de las funciones de la organización y así mismo la generación de información con la existencia de un adecuado sistema de contabilidad, se podrá contribuir a una adecuada toma de decisiones (Di Ranni, 2016).

La contabilidad de gestión brinda información de magnitudes significativas, tales como la eficiencia en la utilización de mano de obra, el costo de los productos, la rentabilidad de cada uno de ellos, entre otros que complementa la información que por su naturaleza no puede brindar la contabilidad financiera, Salvador (1998) indica que muchas veces la contabilidad financiera no le aporta a las empresas la información necesaria y suficiente para la toma de decisiones, 
Atehortúa, T.; Mejía, L. Tipos de decisiones con base en las herramientas de contabilidad...

se convierte enconces la contabilidad de gestión en un proveedor de los indicadores necesarios para la consecución de los objetivos de la empresa.

La relevancia de un sistema integral de información contable se materializa en lo escrito por Chacón (2007), el cual afirma que:

Las organizaciones para alcanzar ventajas competitivas, hoy más que nunca, necesitan una visión sistémica de sí mismas en interacción constante con ese mercado globalizado, estructuras de costos optimizadas, unidades de producción especializadas y la obtención de economías de integración en lugar de economías de escala. De lo que se deduce que deberían contar con un sistema de información contable integral, configurado por una contabilidad financiera y una contabilidad de costos, que alimente a los sistemas de control de gestión y, consecuentemente, permita a sus diferentes usuarios internos beneficiarse de información oportuna, pertinente y comparable a fin de que éstos puedan traducir las estrategias diseñadas para hacer frente al nuevo contexto de los negocios en resultados empresariales. (p. 30)

La contabilidad de gestión le ayuda a la empresa de confecciones a identificar más a profundidad cada una de las actividades en las cuales se invierte el dinero para generar valor, en la determinación clara de sus costos y gastos de forma más técnica y más precisa, también le sirve de gran ayuda a la administración, para que la gerencia optimice sus recursos y utilice herramientas de gestión tanto cualitativas como cuantitativas para la toma de decisiones.

La planeación es decisiva para la toma de decisiones, es por lo que los administrativos la consideran de vital importancia, que bien la define Carreto (2009) como "el arte y ciencia de formular, implantar y evaluar decisiones interfuncionales que permitan a la organización llevar a cabo sus objetivos" (p. 2).

La planeación estratégica provee información confiable, útil y veraz para decidir sobre los objetivos de la empresa, sobre los recursos que se deben utilizar tanto humanos, materiales y financieros.

Cada empresa tiene establecido unos objetivos, por lo tanto es importante hacer uso de una buena planeación, que permita a la entidad aplicar bien los recursos humanos, financieros y materiales, con el fin de cumplir unas metas que le permitan a la empresa su permanencia en el mercado. Para evitar que se despilfarren recursos y se incurra en mayores costos para la empresa, se busca la buena utilización de los materiales, así mismo, para mejorar la calidad de los productos y alcanzar la satisfacción del cliente.

La buena planeación de estos recursos permite a la empresa adelantarse a los acontecimientos de corto y largo plazo, que puedan impedir la consecución de los objetivos tanto estratégicos como operativos, es por esto que el buen control que se hace continuamente a los recursos antes mencionados permite al administrador incrementar las posibilidades de éxito, cumpliendo con los 
objetivos y metas propuestas por la empresa, logrando el buen posicionamiento en el mercado y por consiguiente la consecución de nuevos clientes.

En el municipio de La Ceja la mayoría de MIPYMES de confección operan la contabilidad financiera para la determinación de costos de una manera muy superficial, con poco análisis de la información, se evalúan los costos de manera hipotética y sin tener en cuenta los suficientes datos para hacer un buen costeo de los productos; los procesos productivos en muchas ocasiones no se encuentran organizados ni distribuidos de la mejor manera, lo que hace que constantemente se esté incurriendo en unos mayores costos de fabricación.

El uso de las herramientas de contabilidad de gestión trae una mejor calidad de trabajo para los empleados, el desarrollo regional y mejores beneficios para los clientes que son parte esencial en el crecimiento y mantenimiento de las empresas. Así mismo, se pueden identificar otros beneficios otorgados por la utilización de algunas herramientas de contabilidad de gestión, los cuales se enuncian en la Tabla 2:

Tabla 2. Herramientas de la contabilidad de gestión y sus beneficios

\begin{tabular}{ll}
\hline Herramientas de contabilidad de gestión & Beneficios de su uso en la toma de decisiones \\
\hline \multirow{2}{*}{ Cálculo de costos } & Evaluar la gestión de la empresa \\
& Toma de decisiones operativas y estratégicas \\
& Evaluar resultados traducidos en utilidades \\
Presupuestos & Planificación de actividades \\
& Controlar y evaluar resultados \\
& Toma de decisiones de solución e innovación \\
& Calidad \\
Indicadores no financieros & Tiempos de respuesta efectivos \\
& Reducción de costos \\
& Toma de decisiones operativa y productivas \\
& Visión financiera \\
Modelo Costos-Volumen-Utilidad (CVU) & Comportamiento de los costos \\
& Ayuda en la toma de decisiones \\
& Desarrollo integral de la empresa \\
Balance Scorecard & Gestión de estrategias \\
& Planificar y establecer objetivos \\
& Aumentar el feedback y formación estratégica \\
& Distribuir correctamente costos \\
Costeo Basado en Actividades-ABC & Optimizar actividades dentro de la cadena de valor \\
& Análisis de los beneficios y oportunidades. \\
Cadena De Valor-CDV & Mejorar rentabilidad \\
& Identificación de valor para los clientes \\
& Pensamiento estratégico \\
& Competitividad \\
& Reducción de costos \\
\hline
\end{tabular}

Fuente: elaboración propia a partir de Ripoll y Urquidi (2010), López (2008), López (2005),Lizana (2002) y Quintero y Sanchez (2006). 
Atehortúa, T.; Mejía, L. Tipos de decisiones con base en las herramientas de contabilidad...

\section{Aspectos Metodológicos}

La investigación es de tipo cualitativo debido a que se realizó con un proceso inductivo, es decir, se exploró la situación de las empresas de confección para analizar la interacción de la herramientas de contabilidad de gestión con la toma de decisiones desde un alcance descriptivo especificando cada una de las características y perfiles de las empresas de confección de La Ceja, con el objetivo de determinar el tipo de decisiones que tomaban dichas empresas con base en las herramientas de la contabilidad de gestión.

La metodología empleada se basó en la revisión bibliográfica, aplicando descriptores como herramientas de la contabilidad de gestión y el uso de la contabilidad de gestión en MIPYMES, la contabilidad de gestión en empresas de confección, entre otros. En esta búsqueda de antecedentes de la problemática, se observó que no hay muchas investigaciones de la contabilidad de gestión en el sector objeto de estudio, por lo que se estableció para la selección de los documentos una reciprocidad de investigaciones que evidenciaran el desarrollo de la contabilidad de gestión y las herramientas que permiten que la información obtenida sea pertinente y útil.

Para la consecución del objetivo de la investigación, se desarrolló una encuesta de tipo cualitativo semiestructurada como metodología de exploración para la recolección de información, para posteriormente tratar sus resultados a profundidad.

Las encuestas se realizaron en dos etapas, en la primera se identificó el tipo de empresa, información financiera y administrativa y una conceptualización de funciones del contador y el administrador en la empresa. En la segunda etapa se abordó a profundidad la contabilidad de gestión y las herramientas utilizadas por las empresas en la toma de decisiones; los colaboradores en su mayoría desempeñaban cargos administrativos en las organizaciones lo que permitió obtener información directa del uso de las herramientas y sus beneficios en las decisiones administrativas, productivas y operacionales.

En el municipio de La Ceja hay 32 MIPYMES de las cuales 20 de estas colaboraron brindando la información necesaria para el desarrollo de la investigación, utilizando un muestreo no probabilístico. El número de organizaciones que debían ser abordadas fue determinado en el desarrollo de la investigación, ya que al ser una investigación cualitativa se busca profundidad en la indagación, como es mencionado por Hernández, Fernández y Baptista (2010) "en los estudios cualitativos el tamaño de muestra no es importante desde una perspectiva probabilística, pues el interés del investigador no es generalizar los resultados de su estudio a una población más amplia” (p. 394). 


\section{Análisis de resultados: los tipos de decisiones y su interacción con las herramientas de contabilidad de gestión}

La investigación realizada sirve para mostrar a las empresas del sector de la confección en el municipio de La Ceja, la importancia de la contabilidad de gestión y la utilización de sus herramientas para diferentes tipos de decisiones.

La contabilidad de gestión y la utilización de sus herramientas, permiten a las empresas controlar y maximizar los recursos,obtener eficiencia en sus procesos productivos y tomar decisiones con información no sólo de tipo financiero.

Para que una pequeña empresa permanezca en el tiempo y logre un posicionamiento en el mercado tanto a nivel nacional como internacional, es necesario que maneje muy bien sus procesos internos, por ejemplo: gestión financiera, gestión del talento humano, gestión de producción, gestión de ventas y gestión de compras.

Todo esto se puede lograr a través de la adaptación de un sistema de contabilidad de gestión para la entidad, el cual entre muchos de sus beneficios conllevaría a una mejor calidad de trabajo para sus empleados, entendimiento de las necesidades del cliente y por ende mayor satisfacción de estos, desarrollo regional, entre otros.

Las empresas en el mundo de hoy están obligadas a implementar herramientas de contabilidad de gestión para lograr un mejor desempeño de la organización y obtener un mejor control de cada una de sus operaciones, utilizar la contabilidad de gestión permite mejorar la toma de decisiones, tanto en sus operaciones como en cada uno de los objetivos de la organización (Paz y Dávalos, 2014).

Respecto a los resultados arrojados por el instrumento de investigación se pudo constatar que el $90 \%$ de las empresas ha escuchado sobre la contabilidad de gestión, sin embargo, el $100 \%$ manifestó que han tenido dificultades en su implementación y ejecución, debido a la falta de conocimiento, metodología de trabajo y ausencia de recursos para invertir en ello, dichas limitaciones son atribuidas a la misma organización.

A pesar de todo lo anterior, el 95\% de las empresas respondió que consideran necesaria la contabilidad de gestión para tomar decisiones, mejorar la capacidad de respuesta y solucionar problemas en la organización. Sin embargo, el $85 \%$ manifiesta la necesidad de contar con la colaboración de un profesional externo a la organización para la utilización de la contabilidad de gestión y sus herramientas.

Con base en lo anterior, las compañías rescatan la importancia de la contabilidad de gestión en todos sus procesos a pesar de las dificultades que presentan para su utilización, por lo que sí utilizan herramientas, pero las 
Atehortúa, T.; Mejía, L. Tipos de decisiones con base en las herramientas de contabilidad...

más tradicionales de acuerdo con los resultados obtenidos. De tal manera, las principales decisiones que son apoyadas por las herramientas de la contabilidad de gestión se reflejan en la siguiente gráfica.

Gráfica 1. Tipos de decisiones basadas en herramientas de la contabilidad de gestión

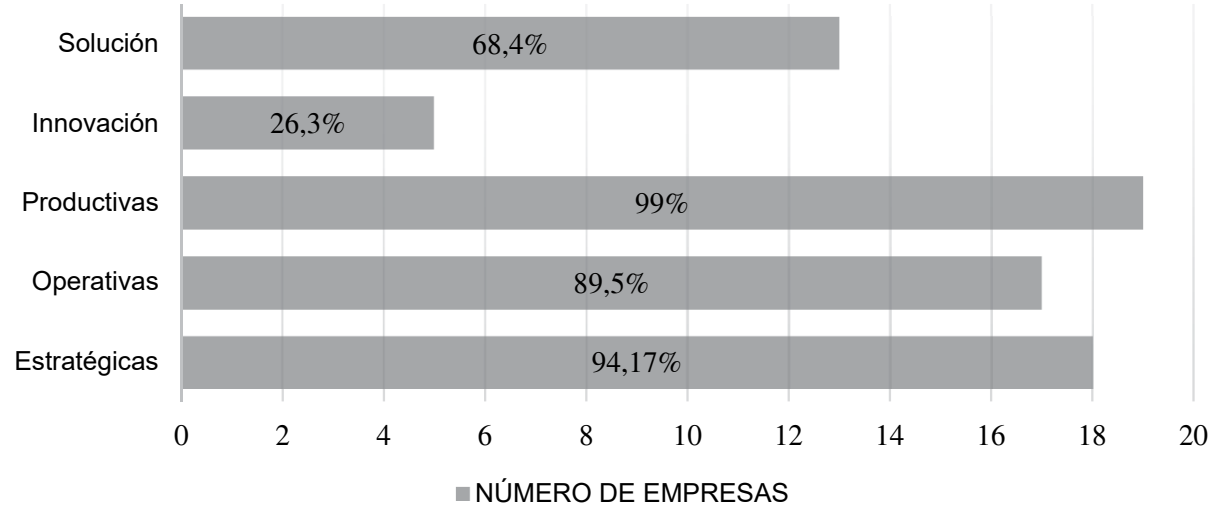

Fuente: Elaboración propia a partir de las entrevistas realizadas

De estas decisiones, la que consideran más importante las organizaciones son las decisiones de tipo operativo. Ahora bien, las decisiones se toman con base en diferentes herramientas que permiten entre tanto, la formulación de objetivos o proyección de la empresa, es decir, las decisiones de solución e innovación, aquellas decisiones con respecto a la medición contable como ingresos, costos, gastos, utilidades y las medidas correctivas para mejorar el funcionamiento de la organización, están traducidas en decisiones estratégicas. Por su parte, el direccionamiento de los recursos y procesos con métodos que se ajusten a las necesidades que surjan en el desarrollo de las actividades y la ejecución de los procesos, se encuentran enmarcadas en las decisiones operativas. Finalmente, y no menos relevante que las anteriores según las encuestas, aquellas que permiten llegar a un nivel de productividad más alto reflejado en la rentabilidad y la sostenibilidad en el mercado son las decisiones productivas.

Las herramientas de contabilidad de gestión utilizadas por las empresas de confección están referenciadas en la siguiente gráfica, en ella se puede apreciar el porcentaje de uso en las empresas encuestadas, lo que permite establecer cuáles de ellas son las que más inciden en la toma de decisiones y en qué tipo específicamente son usadas como se muestra en el siguiente análisis de resultados. 
Gráfica 2. Herramientas de contabilidad de gestión utilizadas

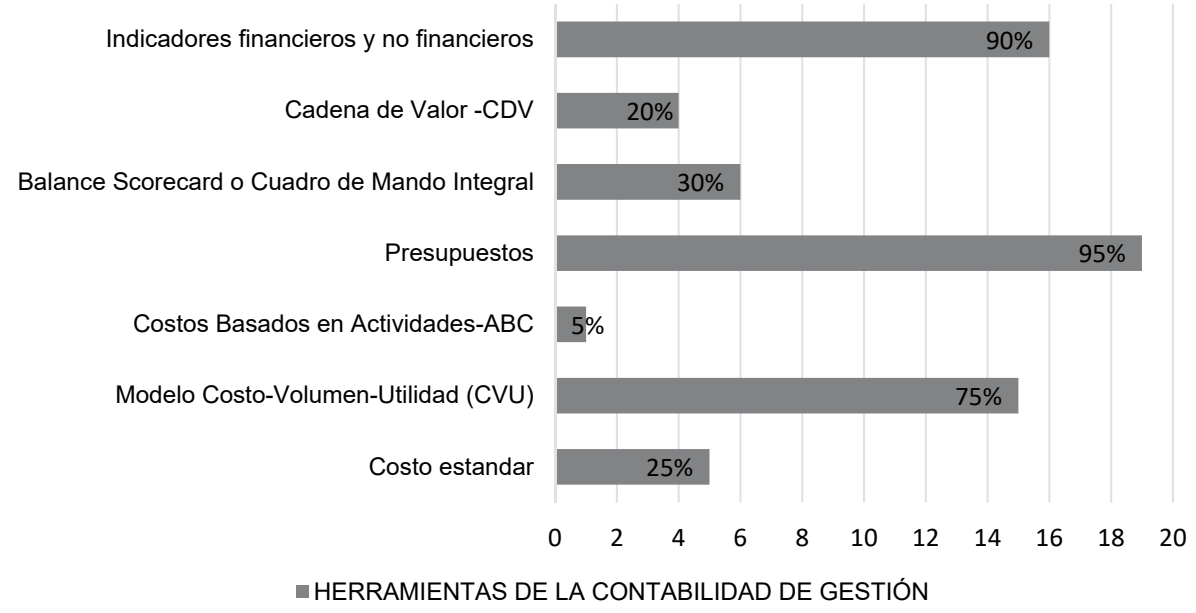

Fuente: elaboración propia basada en los resultados arrojados por las encuestas.

El análisis de los resultados se mostrará mencionando primero el tipo de decisión que se toma y luego, la o las herramientas de contabilidad de gestión en la que se basa, de esta manera se podrá evidenciar la utilización de cada herramienta en las empresas del sector de la confección.

\section{IV.1 Decisiones operativas}

Las decisiones operativas son aquellas que debe tomar la empresa para realizar todas las actividades de producción, es decir aquellas relacionadas directamente con el control de costos y calidad, manufactura del producto y programación del proceso, materiales, maquinaria y recurso humano para la producción.

Dentro de los principios fundamentales para que haya eficiencia en las empresas de confección se encuentra la economía de movimientos, lo que se constituye en un punto clave a la hora de verificar que cada operación se esté realizando en el menor tiempo posible, constantemente se debe estar haciendo seguimiento al personal operativo, para determinar que se está cumpliendo con una tarea asignada en un determinado período de tiempo, de lo contrario se podría estar incurriendo en grandes pérdidas para la empresa.

Es muy importante aclarar que la mano de obra es el elemento principal para la elaboración de prendas de vestir, pues es el encargado de darle la apariencia y modelación necesaria para que la prenda quede en perfectas condiciones y pueda salir al mercado. Siendo la mano de obra un factor imprescindible en el ensamble de prendas, también se convierte en el rubro de mayor participación cuando se va a determinar el costo total del producto. 
Atehortúa, T.; Mejía, L. Tipos de decisiones con base en las herramientas de contabilidad...

Las empresas que fueron objeto de investigación, mencionan dos herramientas de contabilidad de gestión que les permiten medir su eficiencia y productividad, para mejorar la competitividad y enfrentar los retos que exige hoy el mercado mundial. De acuerdo con la consulta acerca de las herramientas de gestión utilizadas, de las 20 empresas objeto de estudio, 16 mencionan utilizar herramientas que se basan en la ingeniería, específicamente en función de estudios de tiempos y secuencia de procesos como se pudo observar en la gráfica 2, la herramienta de indicadores financieros y no financieros.

\section{IV.1.1 Herramientas de contabilidad de gestión: Costos estándar y Reingeniería de Procesos}

La ingeniería, aunque sea un poco ajena a la profesión contable, brinda herramientas importantes para la gestión de las organizaciones, entre las cuales está la reingeniería de procesos y el apoyo en el establecimiento de costos estándar. Si bien la reingeniería de procesos no es una herramienta que estaba mencionada de manera explícita en las encuestas, al momento de revisar la respuesta de las empresas, éstas hacen referencia a los indicadores no financieros como reingeniería de procesos, tales como tiempo de fabricación por prenda, movimientos necesarios en la producción, utensilios necesarios para disminuir tiempos y movimientos, entre otros ligados directamente a la ingeniería industrial que permite la planeación, ejecución y entrega del producto.

Resulta muy necesario para los contadores aliarse con ingenieros de producción, ya que estos brindan la asesoría clave para la optimización de la producción, con el objetivo de reducir tiempos en el área de confección, economizar costos y maximizar su utilidad.

Hoy día el sector de la confección se ha convertido en un mercado de tiempo (minutos), es decir, lo que negocian con los clientes es un valor por minuto y no un producto. Para esto, las empresas realizan una identificación clara de los procesos y actividades necesarias para elaborar un producto, todo con el objetivo de identificar los puntos estratégicos y necesarios que permitan caracterizar los procesos de acuerdo con su nivel de complejidad o facilidad y empezar a determinar o estandarizar tiempos por operación. Por tanto, se deben organizar los productos con un orden lógico, de acuerdo con la secuencia de procesos, para que haya una optimización de tiempo en el momento en que se está elaborando el producto; conforme con esto debe haber una secuencia de operaciones adecuada y una distribución de la maquinaria acorde con los procesos a realizar, dando lugar a la reingeniería de procesos.

Estas empresas al hacer estudios de tiempos y movimientos, les permite conocer el tiempo que tardan en realizar cada actividad, en el desarrollo de un producto, y con este estudio realizan seguimientos individuales y grupales, para 
determinar las causas y consecuencias de las actividades o procesos que están disminuyendo valor al ente, siendo la eficiencia un factor determinante para las empresas de confección, ésta muestra resultados diarios de la capacidad y el ritmo al cual están trabajando cada uno de los operarios.

Para establecer costos estándar, es importante considerar la reingeniería de procesos porque las empresas deben establecer secuencias claras entre un proceso y otro, con el objetivo de optimizar y aprovechar la capacidad de la compañía en lo más que se pueda.

\section{IV.2 Decisiones productivas}

Las decisiones productivas son aquellas que determinan las referencias y cantidad de producción, ya sea por demanda del mercado en general o por solicitud de un cliente específico.

Los costos en las empresas de confección se han convertido en una información transcendental para la elaboración del producto, pues a partir de estos se pueden identificar los métodos y procedimientos que están generando mayor y menor valor en cada uno de los procesos productivos.

En este orden de ideas, es muy importante que las empresas de confección posean adecuadas estructuras de costos en lo que refiere a la diferenciación entre gastos y costos fijos y variables, directos e indirectos para que así las herramientas de contabilidad de gestión posean más información que permita un mejor análisis y toma de decisiones. La característica fundamental de los costos, es que estos son recuperables cuando se venda el producto o servicio y capitalizables al inventario, siempre que este aún no este listo para la venta. Los gastos por el contrario, son desembolsos causados por la administración, distribución y financiación de la empresa, es decir, no tienen que ver directamente con el producto y no se recuperan.

Un factor relevante para determinar dentro de éstas empresas, es la capacidad con que cuenta cada una de las plantas, la capacidad le permite a la empresa comprometerse con un volumen de producción. Además, teniendo como referencia el punto de equilibrio debido a que éste es el punto donde la empresa se puede sostener, sin generar utilidades, ni pérdidas y cuando se llega a ese punto se puede decir, que la empresa ha dado sus primeros pasos para progresar y luego empezar a crecer en un segundo nivel, aumentando sus ingresos de acuerdo con las utilidades deseadas.

Para las empresas que se dedican a maquilar, es fácil hacer una planeación estratégica que les permita llegar al punto de equilibrio, porque son empresas que trabajan para clientes definidos y mantienen producción permanente de acuerdo con su capacidad, la cual está definida en función de los minutos que es capaz de producir. 
Atehortúa, T.; Mejía, L. Tipos de decisiones con base en las herramientas de contabilidad...

Es muy importante que el equipo administrativo de las empresas tenga conocimiento sobre el número de unidades a producir y la cantidad de dinero que deben realizar para llegar a este punto, pues con esa información se pueden hacer comparaciones de los minutos producidos, con la facturación y determinar en qué nivel se encuentran para establecer planes de acción en busca del mejoramiento continuo.

Después de que la empresa supere el punto de equilibrio, debe empezar a planear sus utilidades; esta planeación logrará que la empresa cumpla con objetivos de crecimiento en el corto o mediano plazo, sin embargo, debe existir una buena gestión por parte de la administración, ya que los costos, presupuestos e indicadores, solo muestran cifras para hacer seguimiento constante y mejorar cada día, pero la administración es la encargada de ejecutar para que las metas se cumplan.

\section{IV.2.1 Herramienta de contabilidad de gestión: Modelo Costo-Volumen-}

Utilidad (CVU) con énfasis en el punto de equilibrio

La productividad es el resultado de la combinación entre los ingresos, gastos y costos, para lo que el $75 \%$ de las empresas estudiadas como se puede apreciar en la gráfica 2 utilizan el Modelo Costo-Volumen- Utilidad (CVU). Este modelo se puede considerar como la relación que existe entre la cantidad de bienes y servicios producidos, y la cantidad de recursos variables utilizados en el proceso productivo, en un período de tiempo determinado.

Es una de las herramientas más utilizadas por los administradores para ayudar a determinar la relación que hay entre costo, volumen y utilidad de una empresa, además de ver las maneras como la utilidad puede variar según los cambios realizados en las unidades y precio de venta principalmente. Las empresas utilizan este modelo para tomar decisiones de determinación de precios de venta, planeación de utilidades, entre otros.

El modelo costo-volumen-utilidad, le sirve a la administración para la toma de decisiones en cuanto a:

- Número de unidades a producir para que la empresa no tenga pérdidas.

- Incrementar el nivel de producción de acuerdo con las utilidades que espera obtener la empresa en un período de tiempo determinado.

- Permite que la administración identifique de donde provienen los recursos y cómo se están utilizando dentro de la empresa.

- Brinda la información necesaria para que la administración genere estrategias de sostenibilidad en el mercado.

Para las empresas de confección que se dedican a maquilar a terceros el mercado se ha vuelto muy cambiante, porque hoy no se hacen los negocios con prendas, pues lo que se vende o se compra en este mercado son minutos. Esto, pone a las empresas de confección a trabajar con el precio pactado, por 
lo tanto, se tienen que programar e implementar estrategias para que su valor minuto sea rentable. Dado el caso de que el margen de contribución no alcance los objetivos propuestos, las empresas no tendrán otra opción que empezar a tomar decisiones sobre reducción de costos, porque el precio de venta no lo pueden modificar con sus clientes y es necesario obtener un buen margen de rentabilidad que les permita ser sostenibles en el tiempo. Para la planeación de utilidades es importante analizar las variaciones del modelo costo-volumenutilidad, pues este permite en determinado momento, hacer presupuestos que ayuden a aumentar las utilidades.

\section{IV.3 Decisiones estratégicas}

Las decisiones estratégicas son aquellas que le permiten a la empresa posicionarse en el mercado con el aprovechamiento de los recursos, ofreciendo una mejor calidad. En otras palabras, son aquellas que hacen que la empresa tenga un valor agregado en su cadena de valor, determinan el desarrollo de la empresa ya que relaciona el entorno con la organización.

Las organizaciones de hoy necesitan producir en gran volumen con pocos recursos, de esta manera las empresas aprovechan los recursos con que cuentan al máximo, tratando de eliminar todo el desperdicio posible, por tal motivo realizan análisis de la cantidad de material utilizado en cada proceso, para elaborar el producto y de esta manera evitan despilfarro. La eficacia se puede medir con el costo total del producto, ya que, si este presenta una cifra razonable en su fabricación, se puede llegar a la conclusión de que los recursos se están utilizando convenientemente, de lo contrario la empresa se verá obligada a realizar una reducción de costos para obtener mayor beneficio.

El mercado ahora es más exigente que antes, los consumidores pretenden optimizar su dinero en cada transacción que realizan, por ello, quienes se dedican a desarrollar productos y servicios deben volverse más competitivos y atractivos para contrarrestar la competencia, deben conocer a profundidad el ambiente empresarial en el que se desenvuelven, a fin de mantenerse al día con los cambios del entorno.

Se requiere más calidad a un bajo costo; para que las empresas logren un alto nivel de competitividad, necesitan desarrollar estudios que permitan identificar los costos de la no calidad y con base en esto implementar programas de seguimiento para contrarrestarlos.

\section{IV.3.1 Herramienta del Balance Scorecard}

El Balance Scorecard es utilizado en las empresas de confección en un 30\% como se puede evidenciar en la gráfica 2, permite a las empresas realizar una planeación de la estrategia para llevarla a cabo en su sistema de gestión tomando en cuenta tanto indicadores históricos como futuros, reuniendo así 
Atehortúa, T.; Mejía, L. Tipos de decisiones con base en las herramientas de contabilidad...

objetivos a corto, mediano y largo plazo, para realizar revisiones periódicas y sistemáticas con un feedback a tiempo que les permita aprender y mejorar la estrategia para dar una respuesta más asertiva y rápida en el mercado.

IV.3.2 Herramienta de contabilidad de gestión: modelo Costo-VolumenUtilidad (CVU) con énfasis en el Margen de Contribución Porcentual (MC Porcentual)

El margen de contribución porcentual, representa la diferencia entre el precio de venta y los costos y gastos variables, evidenciando los beneficios que genera una unidad para cubrir los costos y gastos fijos de la empresa.

Para el caso de las organizaciones que se dedican a la maquila, el precio de venta de un minuto es un valor constante que no puede variar, por consiguiente, la administración para tomar decisiones solo puede modificar el costo variable. Si el costo variable aumenta el margen de contribución va a disminuir y el porcentaje que hay que dedicar para los costos y gastos fijos también sería un valor constante mientras estos no se modifiquen, por lo tanto, el valor sacrificado sería la utilidad, mostrando cada vez un menor porcentaje de participación frente al producto. Otra decisión que pueden tomar los administradores con este margen es el aumento en los costos variables, ya sea porque se quiere cambiar las características del producto y esto conlleva a consumir más costos y gastos variables o porque la calidad del producto puede ser mejorada, permitiéndole a la empresa competir con calidad en el mercado, lo que podría generar un aumento en las unidades a vender (volumen de ventas) y por supuesto en las ventas. Con esta variación el margen de contribución disminuye, ya que el precio de venta se mantiene estable.

\section{IV.4 Decisiones de solución e innovación}

Las decisiones de solución e innovación, son aquellas que buscan solucionar los diferentes problemas que se dan en el mercado para no dejar perjudicar los ingresos y a su vez hacen que la empresa no se salga de lo planeado y establecido como objetivo en el desarrollo de su actividad, con soluciones prácticas y que establezcan a la empresa como rentable en el mercado cambiante.

En las empresas de confección los presupuestos tienen una gran importancia, ya que la mayoría manejan esta herramienta para la toma de decisiones, para hacer un presupuesto en estas empresas es importante tener en cuenta las variables macroeconómicas, las cuales en los últimos años han afectado a las empresas grandes de confección de nuestro país, lo que quiere decir que también afecta a las MIPYMES del municipio de La Ceja, esto se debe a los problemas relacionados con los cambios en valor del dólar que perjudica tanto a importaciones como a exportaciones. 
Lo anterior afecta al municipio objeto de investigación puesto que las empresas grandes, las cuales son impactadas por estos cambios económicos, son que le dan el trabajo a estas MIPYMES, de las cuales la mayoría de las encuestadas son maquilas (70\% de las encuestadas). Estos problemas producen también cambios en la microeconomía, donde la empresa mostrará reducción en sus ventas y por ende en sus ingresos, perjudicando a todos sus empleados, ya que habrá despidos por la falta de trabajo, y en muchas situaciones cierre de las empresas, es por lo que deben cuidar los recursos con que cuenta la empresa.

\section{IV.4.1 Herramienta de la contabilidad de gestión: cadena de valor}

Realizada a partir de las actividades estratégicas de la compañía, para comprender y tomar decisiones sobre el funcionamiento de los costos, para generar ventajas competitivas y diferentes soluciones en la cadena productiva, suministros y de distribución.

Es utilizada en las empresas de confección ya que permite identificar y diagnosticar la posición de la empresa respecto a sus competidores, por lo tanto, tomar decisiones de solución e innovación para desarrollar estrategias sostenibles.

\section{IV.4.2 Herramienta de contabilidad de gestión: presupuesto}

Se realiza a partir de la buena planeación que se haga de las metas y objetivos propuestos por una empresa, esto con miras a permanecer en el mercado. Para cumplir con esta filosofía institucional es importante que la empresa sepa en qué sector se encuentra con el fin de encontrar debilidades, oportunidades, fortalezas y amenazas, así como también de la competencia, esto para saber con cuáles estrategias o propuestas puede competir en el mercado (diferenciación, desarrollo del nicho, liderazgo en costo).

Es necesario que las personas que conforman la empresa tengan un sentido de pertenencia por ésta, aportando ideas que le sean útiles para el crecimiento de la misma y por ende de ellos, que son el alma de la empresa; si se tienen en cuenta estos requisitos, el presupuesto de la empresa será un punto de partida para la buena organización de sus operaciones, lo que asegura el éxito de ésta en el mercado.

Realizar un presupuesto ofrece ventajas a las empresas, ya que permite que sus administradores puedan tener una mejor visión para cumplir con los objetivos propuestos, además de que proporciona la habilidad de no derrochar los recursos porque con algo planeado, la responsabilidad de las diferentes áreas de la organización sería controlada de mejor manera. Se debe tener en cuenta que para cumplir estos objetivos se interactúa con todo el personal de la empresa. 
Atehortúa, T.; Mejía, L. Tipos de decisiones con base en las herramientas de contabilidad...

Para minimizar los riesgos que trae consigo el entorno, el 95\% de las empresas analizadas del sector de la confección en el municipio de La Ceja como se aprecia en la gráfica 2, utilizan el Presupuesto Maestro, el cual consiste en una serie de presupuestos que se trabajan por separado, pero que son interdependientes, lo que quiere decir que, si falta alguno o si éste es mal planeado, la cadena que conforma el presupuesto maestro estaría mal hecha, y por consiguiente, se tomarían malas decisiones para el futuro de la empresa.

El presupuesto maestro está compuesto por el presupuesto de ventas, el presupuesto de producción, el presupuesto de materiales directos, el presupuesto de mano de obra directa, el presupuesto de costos indirectos de fabricación, el presupuesto de gastos de ventas y administrativos, el estado de resultados presupuestado y el balance general presupuestado.

Para las empresas de confección son importantes éstas herramientas, ya que así pueden distribuir mejor sus ingresos, en las diferentes actividades de operación, permitiéndoles tener un mejor producto y la satisfacción de los clientes; el presupuesto de ventas es el punto de partida para realizar los otros presupuestos, ya que de este depende el buen funcionamiento de los demás, debido a la generación del ingreso que es el recurso que permite que la empresa pueda operar con mayor tranquilidad.

Las compras de materias primas son necesarias para cumplir con el proceso de producción, razón por la cual deben estar en el momento indicado y con la cantidad precisa cuando se elabora un producto, ya que, si no están, podría haber un cuello de botella y se estancaría la producción, lo que trae consigo el aumento de los costos y además el no cumplimiento con la producción necesaria en el momento oportuno.

En las empresas de confección el presupuesto de mano de obra es uno de los presupuestos más importantes, ya que en su mayoría la herramienta primordial para la transformación del producto son las personas, además es el rubro más representativo frente al costo total, dando a entender que se debe analizar cuidadosamente para que al final del periodo los costos no sean muy elevados y no lleven la empresa a la quiebra.

\section{Conclusiones}

La contabilidad de gestión es poco conocida como línea contable en las empresas de confección del municipio de La Ceja, pues se logró evidenciar que esta es mencionada en el medio, pero no se conoce lo que abarca de manera completa y detallada. Adicionalmente, se encuentra que en las empresas, consideran que la implementación y ejecución de la contabilidad de gestión genera muchas dificultades, debido a la falta de conocimiento, metodología de trabajo y ausencia de recursos para invertir en ello. 
Sin embargo, las herramientas que se logran clasificar dentro de la contabilidad de gestión sí son utilizadas por las organizaciones, considerando que estas son importantes para tomar decisiones, mejorar la capacidad de respuesta y solucionar problemas en las mismas.

Los costos basados en actividades (ABC), la cadena de valor-CDV, el Costo Estándar y el Balance Scorecard son utilizados por menos del $50 \%$ de las empresas encuestadas, mientras que los indicadores financieros y no financieros, los presupuestos y el Modelo Costo Volumen Utilidad - CVU son utilizados por más del $75 \%$ de las compañías. Adicionalmente, se encontró que las empresas utilizan la Reingeniería de Procesos, aunque esta no fue concebida dentro de la encuesta realizada a las mismas.

El modelo Costos Volumen Utilidad (CVU) es utilizado por las empresas encuestadas con el objetivo de determinar la contribución marginal y el punto de equilibrio, pues debido a la alta competitividad en el sector, las empresas deben estar evaluando constantemente el costo de su trabajo para poder determinar el valor mínimo al cual pueden vender y la cantidad de unidades con las cuales no obtendrán pérdidas.

Ahora bien, las decisiones que toman con base en las herramientas de contabilidad de gestión en las empresas objeto de estudio son de tipo operativo, productivo, estratégico, de innovación y de solución. Así, estas diferentes alternativas que emplean las empresas de confección para lograr sus objetivos son oportunas, sin embargo, amerita una adecuada selección de acuerdo con el tipo, naturaleza, tamaño, necesidad, cultura, entre otras, que tenga la compañía.

Las herramientas enmarcadas en la contabilidad de gestión permiten analizar información financiera y no financiera adecuada y óptima para la toma de decisiones, obteniendo análisis más completos respecto a la situación financiera y los resultados de la empresa; el lapso que acontece entre el momento en que se toma la decisión y aquel en que se pueden observar los resultados de la decisión generalmente largo y tiene un impacto significativo en el futuro de la empresa.

El uso de las herramientas permite identificar los costos de producción, establecer estrategias que les permita ser competitivas en el mercado cambiante, tener un control sobre sus procesos y una oportuna planeación de ellos, lo que se verá reflejado en mayor rentabilidad y sostenibilidad en el mercado conforme a las investigaciones existentes en el tema.

Adicionalmente, los beneficios que le brindan las herramientas de contabilidad de gestión a las empresas de confección son por su naturaleza determinantes porque permiten realizar seguimiento, medición y control sobre la organización. El rol de la contabilidad de gestión cobra mucha importancia dadas las necesidades de la organización de controlar cada uno de los 
Atehortúa, T.; Mejía, L. Tipos de decisiones con base en las herramientas de contabilidad...

procesos que brindan información para la toma de decisiones para mejorar el rendimiento organizacional, puesto que los cambios que presenta el mercado han hecho que los sistemas contables de las empresas evolucionen con ellas, lo que implica que las empresas adopten nuevas estructuras de obtención de información más completa.

En términos generales las MIPYMES del área de confección ubicadas en el municipio de La Ceja pueden perfeccionar sus procesos mediante el análisis en su gestión de riesgos enfocados en la productividad y rentabilidad empresarial, gestión humana y de impuestos mediante la relación entre la productividad laboral y el nivel salarial, tanto para los empresarios como para los trabajadores, gestión de proyectos y gestión de efectivo con el incremento de las utilidades mediante el mejoramiento del flujo productivo, gestión del producto mediante producción rápida por medio de la eliminación de aquellas tareas que no produzcan valor y gestión de auditoría con el mejoramiento de la competitividad con una revisión continua del mercado, los cliente y el interior de la compañía.

\section{Referencias Bibliográficas}

Buelvas, C. G., y Mejía Alfaro, G. E. (2015). El papel de la contabilidad de gestión en el sistema de información contable y su incidencia en la rentabilidad de las empresas. Panorama Económico, 22, 91-108.

Carreto, J. (2009). juliocarreto.blogspot.com.co. Recuperado de juliocarreto.blogspot. com.co: http://planeacion-estrategica.blogspot.com.co/

Chacon, G. (2007). La Contabilidad de Costos, los Sistemas de Control de Gestión y la Rentabilidad Empresarial. Actualidad Contable Faces, 10(15), 29-45. Recuperado el febrero de 2018, de http://www.redalyc.org/pdf/257/25701504.pdf

Congreso de la República de Colombia. (2 de Agosto de 2004). Ley 905 del 2004. Recuperado el 11 de 2017, de Secretaría Senado: http://www.secretariasenado.gov. co/senado/basedoc/ley_0905_2004.html

Cuevas, C. (2001). Contabilidad de Costos, enfoque gerencial y de gestión. Bogotá, DC: Pearson Educación de Colombia LTDA.

Di Ranni, M. A. (2016). Biblioteca Digital Universidad de Buenos Aires. Recuperado el 12 de 11 de 2017, de Biblioteca Digital Universidad de Buenos Aires: http://bibliotecadigital. econ.uba.ar/download/tesis/1501-1251_DiRanniMA.pdf

Salvador, E. (1998). Dialnet.com. Recuperadō el 17 de 10 de 2017, de Dialnet.com: https:// dialnet.unirioja.es/descarga/articulo/229732.pdf

Fernández, A. (1994). Contabilidad de gestión en el contexto de la excelencia empresarial. 18(81), 863-886. Recuperado el 3 de 11 de 2017

García, G. D., y Pérez, A. Y. (2015). Universidad Autonoma del Perú. Recuperado el 28 de 10 de 2017, de Universidad Autonoma del Perú: http://repositorio.autonoma.edu.pe/ bitstream/AUTONOMA/146/1/GARCIA\%20-\%20PEREZ.pdf 
Hernández, R., Fernández, C., y Baptista, M. d. (2010). Metodología de la investigación (Quinta ed.). México: Mc Graw Hill.

Lizana, L. (Junio de 2002). Gerencia . Recuperado de Costeo Basado en Actividades: http:// www.emb.cl/gerencia/articulo.mvc?xid $=3117 \mathrm{ysec}=7$

López, R. (2005). Observatorio Iberoamericano. Recuperado de UNA NUEVA VISIÓN DEL BALANCED SCORECARD DESDE LA PERSPECTIVA DEL ENTORNO Y EL MAPA ENTRÓPICO ORGANZIACIONAL: http://www.observatorio-iberoamericano.org/RICG/ N\%C2\%BA_9/Ricardo_L\%C3\%B3pez_P\%C3\%A9rez.pdf

López Rodriguez, M. (2000). Ilustrados.com. Recuperado de Ilustrados.com: http://www. ilustrados.com/tema/6155/Contabilidad-Gestion-evolucionparticularidades.html

López, M.E.(17 de Septiembre de 2008). contabilidad, competitividad y gestión. Recuperado de Modelo costo volumen utilidad: http://contabilidadcompetitividadygestion. blogspot.com/2008/09/modelo-costo-volumen-utilidad.html

Osorio, J. A., Ripoll, V., Álvarez, J., y Atehortúa, T. (2016). Sendas de investigación para la contabilidad de gestión inmersa en el fenómeno del agujero negro. Caminos Contables (Primera ed., págs. 124-153). Medellin, Colombia: Sello Editorial Centro de Investigaciones y Consultorías CIC. Recuperado el 26 de Febrero de 2018

Paz, J. A., y Dávalos, J. M. (2014). Repositorio universidad privada antenor orrego. Recuperado de REPOSITORIO UNIVERSIDAD PRIVADA ANTENOR ORREGO: http:// repositorio.upao.edu.pe/bitstream/upaorep/344/1/PAZ_JORGE_CONTABILIDAD_ GESTION_TOMA_DECISIONES_PARTE1.pdf

Quesada, M. (7 de Noviembre de 2007). Gestiopolis. Recuperado el noviembre de 2017, de Gestiopolis: https:/www.gestiopolis.com/metodologia-abc-abm-empresas-cubanas-apartir-de-estudio-de-variables/

Quintero, J., y Sanchez, J. (2006). La cadena de valor: Una herramienta del pensamiento estratégico. TELOS, 8(3), 377-389. Obtenido de La cadena de valor: Una herramienta del pensamiento estratégico.

Red Cultural del Banco de la República en Colombia. (2000). Sectores económicos. Recuperado de Banrepcultural: http://enciclopedia.banrepcultural.org/index. php?title=Sectores_econ\%C3\%B3micos

Ripoll, V., y Urquidi, A. (2010). Herramientas de la contabilidad de gestión utilizadas en la práctica empresarial: una revisión crítica de los trabajos de investigación. Revista Latinoamericana de Administración, 1-20. 